\title{
Review: high levels of homocysteine are associated with an increased risk for cardiovascular disease
}

Eikelboom JW,Lonn E, Genest JJr, et al. Homocyst(e)ine and cardiovascular disease: a critical review of the epidemiologic evidence. Ann Intern Med 1999 Sep 7;131:363-75.

QUESTIONS: Are high levels of homocysteine associated with an increased risk for cardiovascular disease? Can levels of homocysteine be decreased by folic acid, either alone or combined with vitamin B-6 or B-12?

\section{Data sources}

Epidemiological studies (randomised controlled trials and cohort, case control, and cross sectional studies) were identified by searching Medline (1965 to January 1999) using the terms coronary heart disease, cerebrovascular disease, peripheral vascular disease, and atherosclerosis combined with the terms homocysteine, B-6, B-12, and folic acid. Bibliographies of relevant studies were also checked.

\section{Study selection}

All prospective and retrospective studies were included if they evaluated $>150$ patients who were considered to be cases, if they were published after 1995, and if they measured the association between homocysteine levels and cardiovascular disease or the intake of folic acid or vitamins $\mathrm{B}-6$ and $\mathrm{B}-12$.

\section{Data extraction}

Data were extracted on study design and setting; patient selection criteria, characteristics, and outcomes; homocysteine levels; and use of folic acid and vitamins B-6 and B-12. Data were not combined because of study dissimilarities.

\section{Main results}

Cross sectional and case-control studies showed an association between plasma homocysteine levels and carotid disease (5 studies), coronary disease (2 studies), peripheral vascular disease (1 study), and aortic atherosclerotic disease (1 study). 5 of 7 case-control and cross sectional studies showed an association with increased atherosclerotic vascular disease. 10 of 13 nested case-control studies and 1 cohort study showed an association between high levels of homocysteine and increased cardiovascular disease. A decreased risk for cardiovascular disease was also shown with high levels of folate ( 3 of 5 prospective and 1 of 2 retrospective studies) and vitamin B-6 (2 of 2 prospective and 2 of 2 retrospective studies) but not with high levels of vitamin B-12 (1 prospective and 2 retrospective studies). No randomised controlled trials have evaluated the effect of decreasing homocysteine levels on cardiovascular events, but 9 are under way (>53000 patients are scheduled to be enrolled).

\section{Conclusions}

Epidemiological studies show that high levels of homocysteine are associated with an increased risk for cardio- vascular disease. Some variation across study types exists, with prospective studies showing less consistency of association than retrospective studies.

\section{COMMENTARY}

Eikelboom and colleagues show a relation not only between homocysteine levels and arterial occlusive events but also between high homocysteine levels and low levels of vitamin B-12 and folate. These low levels are higher than levels for classic deficiency (megaloblastic anaemia). The 20\% to 40\% increased risk for occlusive events is similar to the increased risk for coronary artery disease mortality associated with a serum cholesterol level in the range of $7.5 \mathrm{mmol} / \mathrm{l}$ $(240 \mathrm{mg} / \mathrm{dl})$ to $6.2 \mathrm{mmol} / \mathrm{l}(220 \mathrm{mg} / \mathrm{dl}))^{1}$ it is also similar to the increased risk for stroke associated with an increase of about 5 to $6 \mathrm{~mm} \mathrm{Hg}$ in diastolic blood pressure in patients with hypertension.

Causes of high levels of homocysteine include genetic abnormalities for cystathionine synthase (heterozygotes) and methylenetetrahydrofolate reductase, poor nutrition, and probably smoking. In patients with the highest serum homocysteine levels (those homozygous for a defective $\beta$ cystathionine synthase enzyme), venous thrombosis often occurs if other risk factors, such as factor V Leiden or methylenetetrahydrofolate reductase mutations, are present. ${ }^{2.3}$

Supplements of vitamin B-12 or folate, or both, may reduce the level of homocysteine. Folate supplementation has already been shown to decrease rates of neural tube defects in children whose mothers traditionally would not be considered to have folate deficiency. Folate supplements (eg, folic acid, orange juice, liver, kidney, and spinach) are not dangerous or expensive. No trials have shown that reducing levels of homocysteine will reduce cardiovascular events, but many are underway; we may therefore soon know whether daily supplements of folate are beneficial.

Bernard A Cooper, MD Stanford University San Jose, California, USA

1 Levine GN, Keaney JF Jr, Vita JA. Cholesterol reduction in cardiovascular disease. Clinical benefits and possible mechanisms. N Engl J Med 1995;332:512-21.

2 Kluijtmans LA, Boers GH, Verbruggen B, et al. Homozygous cystathionine beta-synthase deficiency, combined with factor V Leiden or thermolabile methylenetetrahydrofolate reductase in the risk of venous thrombosis. Blood 1998;91:2015-18.

3 Ridker PM, Hennekens CH, Lindpaintner K, et al. Mutation in the gene coding for coagulation factor $\mathrm{V}$ and the risk of myocardial infarction, stroke, and venous thrombosis in apparently healthy men. $N$ Engl J Med 1995;332:912-17. 